\begin{tabular}{|c|l|}
\hline Title & $\begin{array}{l}\text { A symmetric synthesis of a diastereomer of the structure proposed for amphidinolide A and the determination of its } \\
\text { absolute configuration }\end{array}$ \\
\hline Author(s) & Ishiyama, Haruaki; Nakamura, Y oichi; Kobay ashi, Jun'ichi \\
\hline Citation & $\begin{array}{l}\text { Tetrahedron, 62(1), 166-170 } \\
\text { https://doi.org/10.1016/.tet.2005.09.120 }\end{array}$ \\
\hline Issue Date & 2005-10-25 \\
\hline Doc URL & http://hdl.handle.net/21159966 \\
\hline Type & article (author version) \\
\hline File Information & TET62-1.pdf \\
\hline
\end{tabular}

Instructions for use 


\title{
Asymmetric Synthesis of a Diastereomer of the Structure Proposed for Amphidinolide A and the Determination of Its Absolute Configuration
}

\author{
Haruaki Ishiyama, Yoichi Nakamura, and Jun’ichi Kobayashi* \\ Graduate School of Pharmaceutical Sciences, Hokkaido University, Sapporo 060-0812, Japan
}

Abstract An asymmetric synthesis of a diastereomer (2) of the structure (1) proposed for amphidinolide A, a cytotoxic macrolide from the cultured dinoflagellate Amphidinium sp., has been accomplished. The absolute configuration of amphidinolide A was established as $\mathbf{3}$ from comparison of NMR data, HPLC analysis, and $[\alpha]_{D}$ values of amphidinolide $A$, and comparison with the synthetic diastereomers $\mathbf{2}$ and 3, the latter of which was synthesized previously by Trost's group.

Keywords: Amphidinium sp.; macrolide; amphidinolide A; absolute configuration

Amphidinolide A is a cytotoxic 20-membered macrolide, isolated from the cultured dinoflagellate Amphidinium sp., which is a symbiont of the Okinawan marine flatworm Amphiscolops sp. ${ }^{1 \mathrm{a}}$ The relative stereochemistry of the nine stereogenic centers in amphidinolide A was proposed to be 1 on the basis of extensive NMR experiments by our group. ${ }^{1 b}$ The unique structure and bioactivity of amphidinolide A have prompted studies of its total syntheses. $\quad$ First Pattenden, $^{2}$ and later Maleczka, ${ }^{3}$

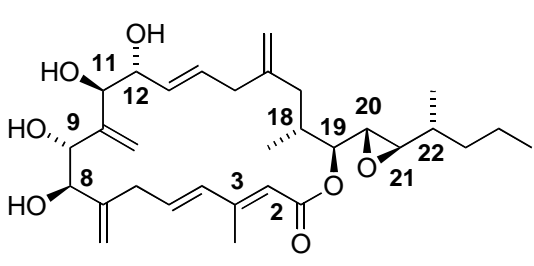

1

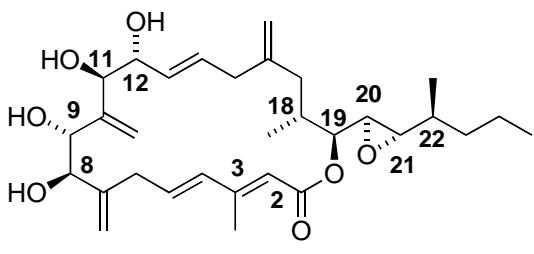

2

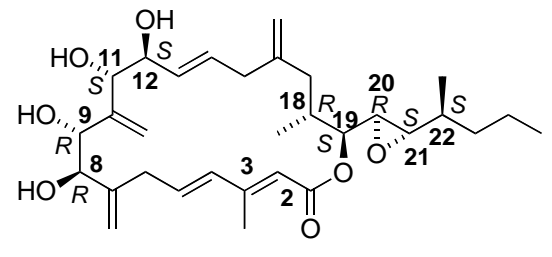

3

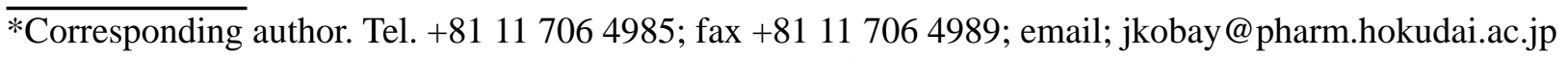


and Trost ${ }^{4}$ accomplished total syntheses of the stereostructure (1) proposed for amphidinolide A, and indicated that the proposed stereostructure (1) was incorrect from comparison of the NMR data of their synthetic compounds with those reported for amphidinolide A. More recently, Trost's group achieved the syntheses of nine stereoisomers of the proposed stereostructure (1), and suggested that the diastereomer 3 may be the correct stereostructure of amphidinolide A.4b,c,d

In our efforts to determine the correct stereostructure of amphidinolide A, we have re-examined the relative stereochemistry of amphidinolide A. Our re-examination of the ${ }^{1} \mathrm{H}$ and ${ }^{13} \mathrm{C}$ NMR data have indicated that the correct stereostructure of amphidinolide A could be either of the diastereomer $\mathbf{2}$ or $\mathbf{3}$. Since the diastereomer $\mathbf{3}$ has been synthesized by Trost's group, ${ }^{4 b}$ we decided to synthesize the alternative diastereomer $\mathbf{2}$ and to compare the NMR data of the synthetic diastereomers $\mathbf{2}$ and $\mathbf{3}$ with those of naturally derived amphidinolide A. In this paper, we describe an asymmetric synthesis of the diastereomer $\mathbf{2}$, and the determination of the absolute stereochemistry of amphidinolide A to be $\mathbf{3}$.

Although earlier we reported previously the relative stereochemistry for C-19 and C-20 in narural amphidinolide A as 19,20-threo from NOESY correlations of $\mathrm{H}-19$ to $\mathrm{H}-21, \mathrm{H}-20$ to $\mathrm{H}_{2}-17$, and H-20 to $\mathrm{H}_{3}$-30 (Figure 1(a)), the proposed stereostructure 1 was not correct. Alternatively, it was

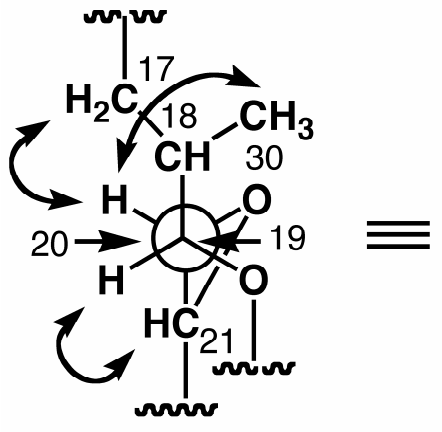

(a)

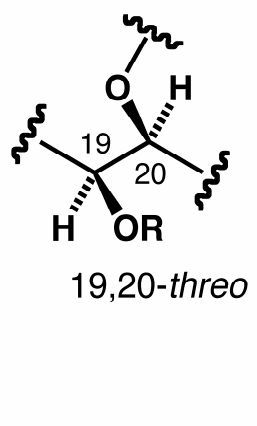

)

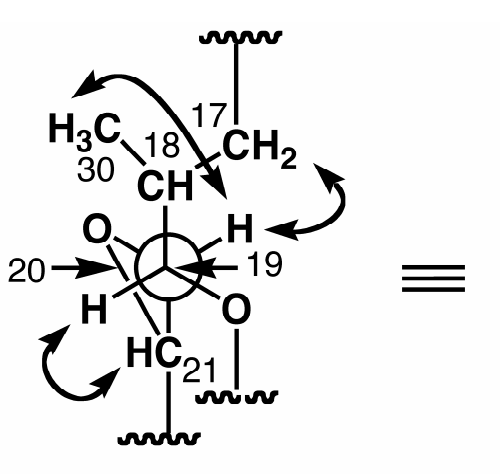

(b)

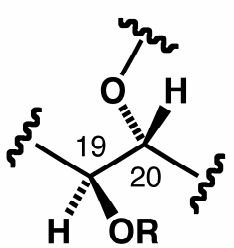

19,20-erythro

${ }^{3} J_{\mathrm{H} 19 / \mathrm{H} 20}=6.8 \mathrm{~Hz}$

Figure 1. NOESY correlations and relative stereochemistry for C-17 C-21 segments in amphidinolide A based on the previous (a) and present (b) assignments. 
elucidated to be 19,20 -erythro from the NOESY correlations and the ${ }^{1}{ }^{\mathrm{H}-}{ }^{1} \mathrm{H}$ coupling constant $(6.8 \mathrm{~Hz})$ between H-19 and H-20 (Figure 1(b)). On the other hand, the ${ }^{13} \mathrm{C}$ NMR data obtained for amphidinolide A were compared with those of the synthetic compound $\mathbf{1}$ reported by the Maleckzka group, ${ }^{3}$ in which the difference $(\Delta+1.8 \mathrm{ppm})$ in chemical shifts at C-12 was slightly larger than those $(|\Delta|<1.3 \mathrm{ppm})$ of the other stereogenic centers. ${ }^{5}$ The ${ }^{1} \mathrm{H}-{ }^{1} \mathrm{H}$ coupling constant $(\sim 0 \mathrm{~Hz})$ between $\mathrm{H}-11$ and H-12 indicated 11,12-threo as reported previously. ${ }^{1 \mathrm{~b}}$ These observations suggested that the possible stereostructure of amphidinolide A could be either diastereomer $\mathbf{2}$ or $\mathbf{3}$.

Since Trost's group has recently reported a synthesis of the diastereomer $\mathbf{3}$, we planned to synthesize the other possible diastereomer 2, using the synthetic strategy by Trost ${ }^{4 b}$, but using Kita's esterification 6 and a ruthenium-catalyzed coupling reaction ${ }^{7}$ (Scheme 1).

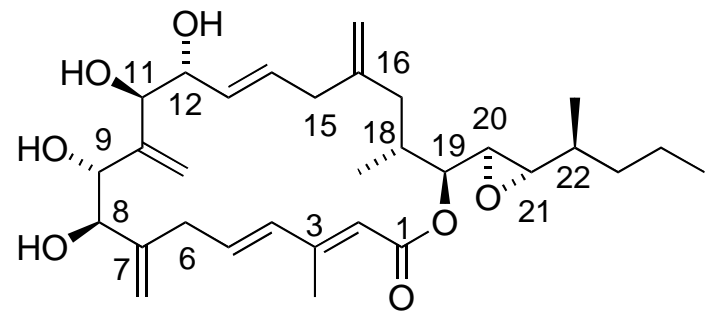

2

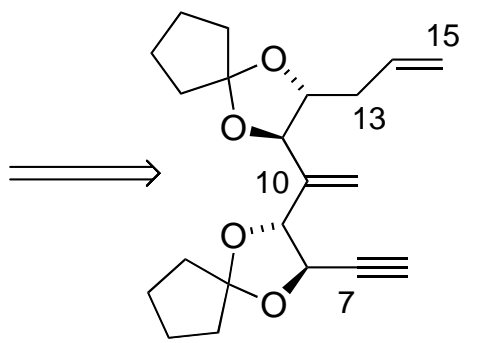

5

\section{Scheme 1}

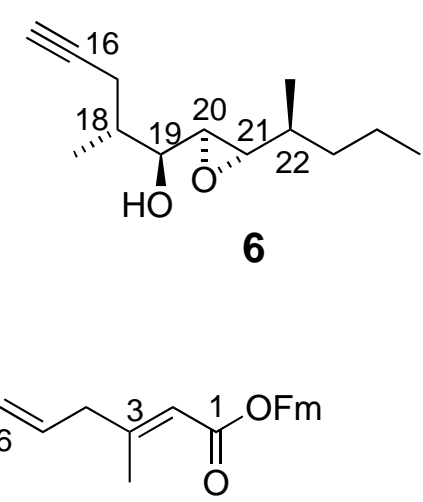

4

The alkyne $5^{4 \mathrm{~b}}$ and the alkene $4^{4 \mathrm{~b}}$ were prepared according to the same procedure as Trost's group. Treatment of the alkyne $\mathbf{5}$ and the alkene $\mathbf{4}$ with ruthenium catalyst provided $\mathbf{7}$ and its isomer $\mathbf{7}$ ' (Scheme 2). After deprotection of the fluorenymethanol (Fm) group in 7 with piperidine, the protecting group of $\mathbf{8}$ was changed from ketal to TES ether to give the acid $\mathbf{9} .^{8}$ Esterification of $\mathbf{9}$ with the alchol $\mathbf{6}$ using the modified method ${ }^{9}$ of Kita $^{6}$ provided the desired ester $\mathbf{1 0}$ with no isomerization of any olefin moiety. ${ }^{10}$ After removal of the TES group, intramolecular cycloisomerization of 11 under high dilution conditions, to form the C15-C16 bond, gave the desired diastereomer 2. 
$\mathrm{x}$

Scheme 2.

The ${ }^{1} \mathrm{H}$ and ${ }^{13} \mathrm{C}$ NMR data for synthetic compound 2 were not coincident with those of amphidinolide A, whereas the NMR data for compound 3 synthesized by Trost's group were close to those of amphidinolide A (Figure 2). Compounds 2 and 3, and amphidinolide A were subjected to $\mathrm{C}_{18}$ HPLC [Mightysil RP-18, 4.6 x $250 \mathrm{~mm}$; flow rate $1.0 \mathrm{~mL} / \mathrm{min}$ : eluent; $\mathrm{MeCN} / \mathrm{H}_{2} \mathrm{O}$ (60:40); UV detection at $265 \mathrm{~nm}$ ], and it was found that the retention time of amphidinolide $\mathrm{A}\left(\mathrm{t}_{R} 16.5 \mathrm{mim}\right)$ was identical with that of $3\left(\mathrm{t}_{R} 16.5 \mathrm{~min}\right)$ but not that of $\mathbf{2}\left(\mathrm{t}_{R} 12.8 \mathrm{~min}\right)$. Thus, the relative stereostructure for amphidinolide A was assigned as $\mathbf{3}$. The optical rotations of compounds $\mathbf{2}$ and $\mathbf{3}$, and amphidinolide A were compared as follows; $[\alpha]_{\mathrm{D}}^{21}-11^{\circ}\left(c 0.6, \mathrm{CHCl}_{3}\right)$ for $2,[\alpha]_{\mathrm{D}}{ }^{24}+56^{\circ}\left(c 0.05, \mathrm{CHCl}_{3}\right)$ for 3 , and $[\alpha]_{\mathrm{D}}{ }^{24}+46^{\circ}\left(c\right.$ 1.0, $\left.\mathrm{CHCl}_{3}\right)$ for amphidinolide A. Therefore, it was concluded that the absolute 
configurations at the nine chiral centers of amphidinolide A were $8 R, 9 R, 11 S, 12 S, 18 R, 19 S, 20 R, 21 S$, and 22S.

(a)

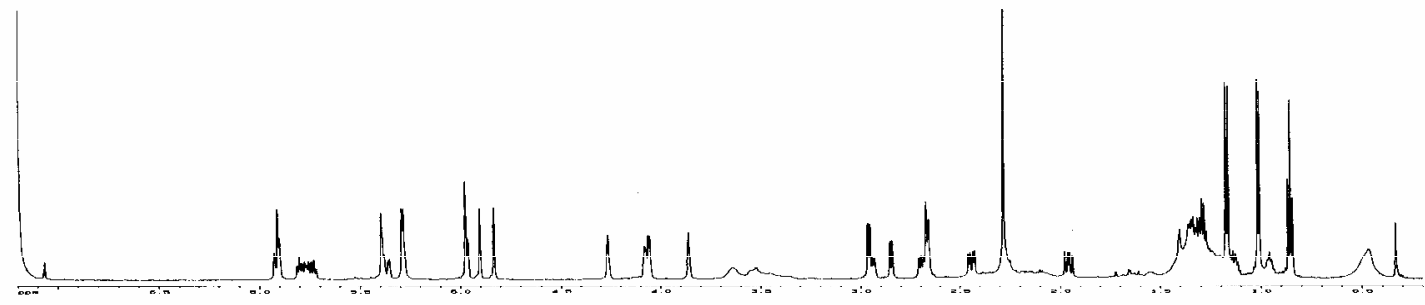

(b)

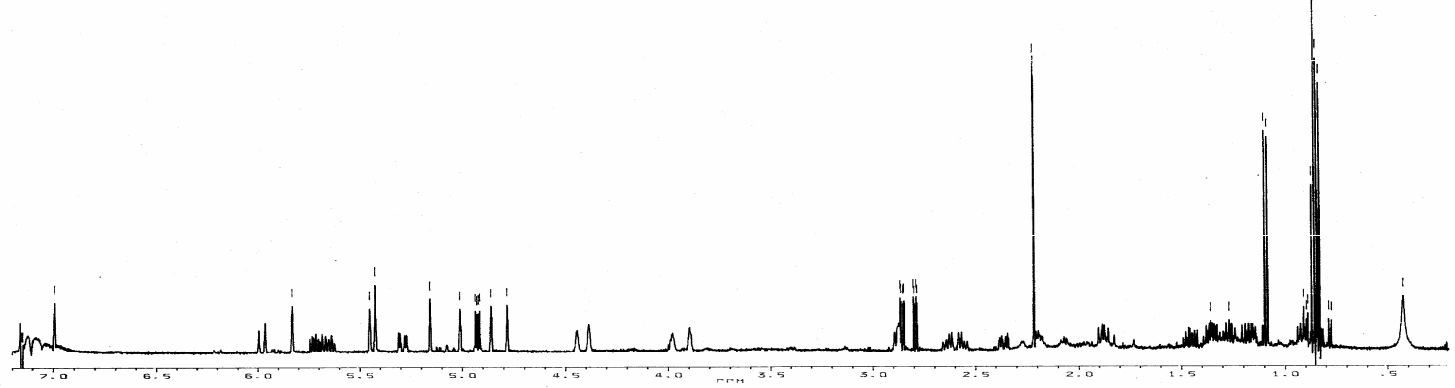

(c)

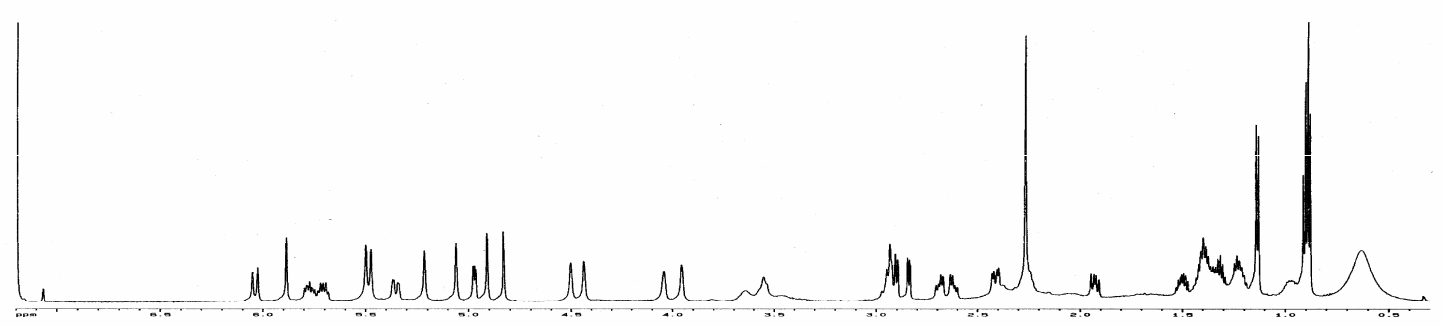

Figure 2. ${ }^{1} \mathrm{H}$ NMR profiles of synthetic diastereomers 2 (a) and 3 (c), and amphidinolide $A(b)$ in $\mathrm{C}_{6} \mathrm{D}_{6}$.

(a)

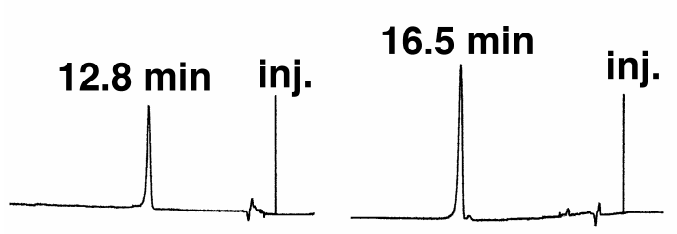

(c)

(d)

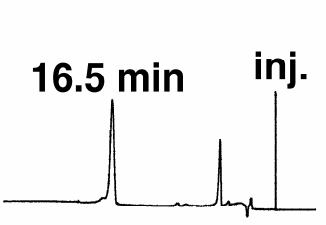

$16.5 \mathrm{~min}$

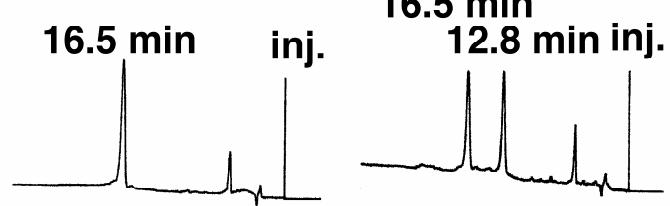

Figure 3. HPLC profiles of synthetic diastereomers 2 (a) and 3 (b), amphidinolide A (c), 3 and amphidinolide A (d), 2 and amphidinolide A (e) 


\section{Experimental Section}

General Methods. Optical rotations were recorded on a JASCO DIP-1000 polarimeter. The IR spectrum was taken on a JASCO FT/IR-5300 spectrometer. Proton and carbon NMR spectra were recorded on a Bruker 500 and/or $600 \mathrm{MHz}$ and JEOL $400 \mathrm{MHz}$ spectrometer. ESI mass spectra were obtained on a JEOL JMS-SX102A spectrometer.

\section{7-\{3-[1-(3-Allyl-1,4-dioxa-spiro[4,4]non-2-yl)-vinyl]-1,4-dioxa-spiro[4,4]non-2-yl\}-3-methyl-octa-2,}

\section{4,7-trienoic acid 9 H-fluoren-9-ylmethyl ester (7)}

A solution of alkyne $5^{4 \mathrm{a}}(208.4 \mathrm{mg}, 0.605 \mathrm{mmol})$ and alkene $4^{4 \mathrm{a}}(916.5 \mathrm{mg}, 3.01 \mathrm{mmol})$ in dichloroethane (DCE) (1.7 mL) was degassed by F. T. P. (Freeze-pump-thaw cycles), the reaction mixture was heated to $50^{\circ} \mathrm{C}$, and $\mathrm{Cp} * \mathrm{Ru}(\mathrm{MeCN}){ }_{3} \mathrm{PF}_{6}(60.7 \mathrm{mg}, 0.120 \mathrm{mmol})$ was added in one portion. After $3 \mathrm{~h}$, the reaction mixture was purified by flash column chromatography on silica gel (10\% to 15\% $\mathrm{Et}_{2} \mathrm{O}$ in petroleum ether) to give branched ester 7 (141.4 mg, $\left.0.218 \mathrm{mmol}, 36 \%\right)$ and linear ester 7' (47.3 mg, $0.0729 \mathrm{mmol}, 12 \%)$ as colorless oils. Data for branched alkene 7: ${ }^{1} \mathrm{H}$ NMR (400 $\left.\mathrm{MHz}, \mathrm{CDCl}_{3}\right) \delta$ 7.77 (d, $J=7.6 \mathrm{~Hz}, 2 \mathrm{H}$ ), 7.62 (d, $J=8.0 \mathrm{~Hz}, 2 \mathrm{H}$ ), 7.41 (dd, $J=7.6,7.6 \mathrm{~Hz}, 2 \mathrm{H}$ ), 7.32 (dd, $J=8.0,7.6$ Hz, 2 H), 6.16-6.23 (m, 2 H), 5.81-5.89 (m, 2 H), 5.42-5.49 (m, 2 H), 5.24 (s, 1 H), 5.09-5.14 (m, 2 H), 5.01 (s, $1 \mathrm{H}), 4.39-4.43$ (m, 3H), 4.26 (t, $J=7.2 \mathrm{~Hz}, 1 \mathrm{H}), 4.20$ (d, $J=8.8 \mathrm{~Hz}, 1 \mathrm{H}), 4.06$ (d, $J=8.0 \mathrm{~Hz}$, $1 \mathrm{H}$ ), 3.89-3.94 (m, $1 \mathrm{H}), 3.05$ (dd, $J=16.4,5.6 \mathrm{~Hz}, 1 \mathrm{H}), 2.92$ (m, $1 \mathrm{H}), 2.43$ (m, $1 \mathrm{H}), 2.25-2.31$ (m, 4H), 1.67-1.90 (m, $16 \mathrm{H})$; $\left.{ }^{13} \mathrm{C} \mathrm{NMR} \mathrm{(100} \mathrm{MHz,} \mathrm{CDCl}_{3}\right)$ 166.8, 152.7, 143.9, 143.3, 142.4, 141.2, 135.6, 134.1, 133.9, 127.6, 127.0, 125.0, 119.9, 119.0, 118.6, 117.9, 117.7, 117.4, 115.6, 84.0, 81.3, 79.8, 79.7, 65.8, 46.9, 37.7, 37.5, 37.4, 37.4, 36.4, 34.7, 23.6, 23.5, 23.5, 14.0; ESIMS m/z $480\left(\mathrm{M}-\mathrm{C}_{10} \mathrm{H}_{16} \mathrm{O}_{2}\right)$.

\section{7-\{3-[1-(3-Allyl-1,4-dioxa-spiro[4,4]non-2-yl)-vinyl]-1,4-dioxa-spiro[4,4]non-2-yl\}-3-methyl-octa-2,}

\section{4,7-trienoic acid (8)}

To a solution of branched ester $7(170.3 \mathrm{mg}, 0.262 \mathrm{mmol})$ in $\mathrm{CH}_{2} \mathrm{Cl}_{2}(6.9 \mathrm{~mL})$ at $0{ }^{\circ} \mathrm{C}$ was added piperidine $(0.86 \mathrm{~mL}, 8.7 \mathrm{mmol})$. After $2.5 \mathrm{~h}$ at room temperature, the reaction mixture was diluted 
with $\mathrm{CH}_{2} \mathrm{Cl}_{2}$ (30 mL), washed with $0.2 \mathrm{M} \mathrm{H}_{2} \mathrm{SO}_{4}$ (x 3), brine, dried over $\mathrm{MgSO}_{4}$, and concentrated in vacuo. Purification by flash column chromatography on silica gel (hexane/EtOAc/MeOH, 30:1:2) gave acid 8 (111.2 mg, 90\%) as a colorless oil.

8: ${ }^{1} \mathrm{H}$ NMR (400 MHz, $\left.\mathrm{CDCl}_{3}\right) \delta$ 6.14-6.20 (m, $\left.2 \mathrm{H}\right)$, 5.80-5.90 (m, $\left.1 \mathrm{H}\right), 5.75$ (s, $\left.1 \mathrm{H}\right), 5.47$ (s, $1 \mathrm{H}$ ), 5.46 (s, $1 \mathrm{H}), 5.23$ (s, $1 \mathrm{H}), 5.09-5.13$ (m, $2 \mathrm{H}), 4.99$ (s, $1 \mathrm{H}), 4.40$ (d, $J=8.4 \mathrm{~Hz}, 1 \mathrm{H}), 4.18$ (d, $J=8.4$ Hz, 1 H), 4.05 (d, $J=8.0$ Hz, 1 H), 3.90 (td, $J=7.4,4.0$ Hz, 1 H), 3.02-3.07 (m, $1 \mathrm{H}), 2.87-2.92$ (m, 1 H), 2.38-2.43 (m, $1 \mathrm{H}), 2.30$ (s, $3 \mathrm{H}), 2.24-2.33$ (m, 1H), 1.64-1.91 (m, $17 \mathrm{H})$; ${ }^{13} \mathrm{C}$ NMR (100 MHz, $\left.\mathrm{CDCl}_{3}\right) \delta 171.9,154.4,143.2,142.3,135.5,134.7,133.8,119.0,118.6,117.7,117.6,117.3,115.6,84.0$, 81.2, 79.7, 79.7, 37.6, 37.5, 37.4, 37.4, 36.3, 34.7, 23.6, 23.5, 23.5, 14.2; ESIMS m/z $493(\mathrm{M}+\mathrm{Na})$;

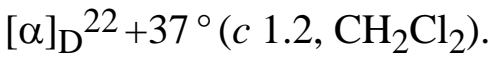

\section{3-Methyl-7,10-dimethylene-8,9,11,12-tetrakis-triethylsilanyloxy-pentadeca-2,4,14-trienoic acid (9)}

To ketal 8 (111.2 mg, $0.236 \mathrm{mmol})$ at room temperature was added acetic acid (1.5 mL) and water (0.5 $\mathrm{mL}$ ). The reaction mixture was heated to $40{ }^{\circ} \mathrm{C}$ for $24 \mathrm{~h}$ and concentrated to give the tetraol which was used in the next step without further purification. To a solution of the tetraol in THF $(5.9 \mathrm{~mL})$ at $0{ }^{\circ} \mathrm{C}$ was added $i$-Pr2NEt (576 $\mu \mathrm{L}, 3.31 \mathrm{mmol})$ and TESOTf $(530 \mu \mathrm{L}, 2.36 \mathrm{mmol})$. The reaction mixture was stirred at $0{ }^{\circ} \mathrm{C}$ for $20 \mathrm{~min}$, quenched with $1 \mathrm{M} \mathrm{HCl}$ (3.3 mL), stirred for $10 \mathrm{~min}$, and diluted with ether (3 $\mathrm{mL})$ and water $(3 \mathrm{~mL})$. The aqueous phase was extracted with ether $(3 \mathrm{x})$ and the combined organic extracts were washed with saturated $\mathrm{KH}_{2} \mathrm{PO}_{4}(1 \mathrm{x})$, brine $(1 \mathrm{x})$, dried over $\mathrm{MgSO}_{4}$, and concentrated. Purification by flash column chromatography on silica gel (9\% EtOAc in hexanes) gave silyl ether 9 (172.9 mg, 92\%) as a colorless oil

9: ${ }^{1} \mathrm{H}$ NMR (500 MHz, $\left.\mathrm{CDCl}_{3}\right) \delta$ 6.11-6.20 (m, $\left.2 \mathrm{H}\right)$, 5.80-5.85 (m, $\left.1 \mathrm{H}\right), 5.74$ (s, $\left.1 \mathrm{H}\right), 5.32$ (s, $2 \mathrm{H}$ ), 
4.96-5.03 (m, 3 H), 4.84 (s, 1 H), 4.49 (d, $J=4.0 \mathrm{~Hz}, 1 \mathrm{H}), 4.35$ (d, $J=3.5 \mathrm{~Hz}, 1 \mathrm{H}), 4.15$ (d, $J=4.0 \mathrm{~Hz}$, 1 H), 3.74 (ddd, $J=8.2,4.0,3.5 \mathrm{~Hz}, 1 \mathrm{H}$ ), 3.01 (dd, $J=16.3,5.5 \mathrm{~Hz}, 1 \mathrm{H}), 2.94$ (dd, $J=16.3,6.5 \mathrm{~Hz}, 1$ H), 2.34-2.40 (m, 2 H), 2.30 (s, 3 H), 0.90-0.99 (m, 36 H), 0.56-0.67 (m, $24 \mathrm{H})$; ${ }^{13} \mathrm{C}$ NMR (125 MHz, $\left.\mathrm{CDCl}_{3}\right) \quad 171.9,155.1,148.4,146.9,137.1,136.3,135.0,116.9,116.1,113.6,113.5,80.1,76.8,74.9$, 74.7, 36.9, 36.6, 14.0, 7.1, 7.1, 7.0, 7.0, 5.5, 5.2, 4.9, 4.9; IR (film) $v_{\max } 2955,2874,1686,1609,1092$, and $742 \mathrm{~cm}^{-1}$; HRESIMS calcd for $\mathrm{C}_{42} \mathrm{H}_{82} \mathrm{O}_{6} \mathrm{Si}_{4} \mathrm{Na} \mathrm{m} / \mathrm{z}$ 817.5086, found $\mathrm{m} / \mathrm{z}$ 817.5078; $[\alpha]_{\mathrm{D}}{ }^{22}+29^{\circ}(\mathrm{c}$ $\left.1.0, \mathrm{CHCl}_{3}\right)$.

\section{3-Methyl-7,10-dimethylene-8,9,11,12-tetrakis-triethylsilanyloxy-pentadeca-2,4,14-trienoic}

\section{2-methyl-1-[3-(1-methyl-butyl)-oxiranyl]-pent-4-ynyl ester (10)}

To a solution of acid $9(17.7 \mathrm{mg}, 22.3 \mu \mathrm{mol})$ in toluene $(0.59 \mathrm{~mL})$ at room temperature was added $\left[\operatorname{RuCl}_{2}(p \text {-cymene })\right]_{2}(1.4 \mathrm{mg}, 17.7 \mu \mathrm{mol})$ and a toluene $(0.19 \mathrm{~mL})$ solution of ethyl ethynyl ether (40 wt $\%$ in hexane, $16 \mu \mathrm{L}, 66.8 \mu \mathrm{mol})$. The reaction mixture was stirred at room temperature for $3 \mathrm{~h}$ and concentrated under a stream of argon. A solution of epoxy alcohol 6 (11.7 mg, $55.6 \mu \mathrm{mol})$ in DCE (0.15 mL) was added via cannula followed by CSA $(0.52 \mathrm{mg}, 2.24 \mu \mathrm{mol})$ and MS3A (10 mg). The reaction mixture was stirred at room temperature for $2 \mathrm{~h}$, filtered through silica gel, and concentrated. Purification by flash column chromatography on silica gel (5\% EtOAc in hexane) gave ester 10 (9.3 mg, $42 \%)$ as a colorless oil.

10: ${ }^{1} \mathrm{H}$ NMR (600 MHz, $\mathrm{C}_{6} \mathrm{D}_{6}$ ) $\delta$ 6.23-6.30 (m, $2 \mathrm{H}$ ), 6.07 (dddd, $J=17.0,9.4,7.7,7.2 \mathrm{~Hz}, 1 \mathrm{H}$ ), 5.89 (s, $1 \mathrm{H}), 5.70$ (s, $1 \mathrm{H}), 5.67$ (s, $1 \mathrm{H}), 5.31$ (s, $1 \mathrm{H}), 5.25$ (d, $J=17.0 \mathrm{~Hz}, 1 \mathrm{H}$ ), 5.15 (d, $J=9.4 \mathrm{~Hz}, 1 \mathrm{H}), 5.07$ (s, $1 \mathrm{H}), 4.95$ (dd, $J=7.1,4.6 \mathrm{~Hz}, 1 \mathrm{H}), 4.78$ (d, $J=3.8 \mathrm{~Hz}, 1 \mathrm{H}), 4.62$ (d, $J=2.8 \mathrm{~Hz}, 1 \mathrm{H}), 4.41$ (d, $J=$ $3.8 \mathrm{~Hz}, 1 \mathrm{H}$ ), 4.02 (ddd, $J=8.2,3.9,2.8 \mathrm{~Hz}, 1 \mathrm{H}$ ), 3.16-3.23 (m, $2 \mathrm{H}$ ), 2.89 (dd, $J=7.6,1.7 \mathrm{~Hz}, 1 \mathrm{H}$ ), 
2.75 (dd, $J=7.1,1.7$ Hz, 1 H), 2.70-2.73 (m, 1 H), 2.47 (s, 3 H), 2.33-2.40 (m, 3 H), 2.12-2.20 (m, 1 H), 1.82 (t, $J=2.3 \mathrm{~Hz}, 1 \mathrm{H}), 1.18$ (d, $J=6.5 \mathrm{~Hz}, 3 \mathrm{H}), 1.09-1.32(\mathrm{~m}, 41 \mathrm{H}), 0.99$ (d, $J=6.6 \mathrm{~Hz}, 3 \mathrm{H})$, 0.74-0.87 (m, $27 \mathrm{H}) ;{ }^{13} \mathrm{C}$ NMR (150 MHz, $\left.\mathrm{C}_{6} \mathrm{D}_{6}\right) \delta 165.9,153.4,149.0,147.5,137.2,135.8,135.2$, 118.1, 116.7, 114.2, 113.9, 82.3, 80.7, 77.4, 75.4, 75.3, 75.1, 70.2, 62.8, 57.0, 37.5, 37.2, 36.1, 35.9, 35.7, 22.7, 20.5, 17.3, 14.5, 14.4, 14.0, 7.5, 7.4, 7.4, 7.3, 6.0, 5.7, 5.5, 5.4; IR (neat) $v_{\max } 2956,2121,1717$, 1235, $1146 \mathrm{~cm}^{-1}$; HRESIMS calcd for $\mathrm{C}_{55} \mathrm{H}_{102} \mathrm{O}_{7} \mathrm{Si}_{4} \mathrm{Na} \mathrm{m} / \mathrm{z}$ 1009.6600, found $\mathrm{m} / \mathrm{z}$ 1009.6638; $[\alpha]_{\mathrm{D}}{ }^{20}$ $+41^{\circ}\left(\right.$ c $\left.1.0, \mathrm{CH}_{2} \mathrm{Cl}_{2}\right)$.

\section{8,9,11,12-Tetrahydroxy-3-methyl-7,10-dimethylene-pentadeca-2,4,14-trienoic}

\section{2-methyl-1-[3-(1-methyl-butyl)-oxiranyl]-pent-4-ynyl ester (11)}

To a solution of silyl ether $10(9.3 \mathrm{mg}, 9.42 \mu \mathrm{mol})$ in THF $(0.2 \mathrm{~mL})$ at $0{ }^{\circ} \mathrm{C}$ was added a mixture of TBAF $(190 \mu \mathrm{L}, 1 \mathrm{M}$ in THF, $190 \mu \mathrm{mol})$ and acetic acid $(21.5 \mu \mathrm{L})$. The reaction mixture was stirred at room temperature for $24 \mathrm{~h}$, diluted with water (3 mL), extracted with EtOAc (4 mL x 3), washed with brine, dried over $\mathrm{MgSO}_{4}$, and concentrated in vacuo. Purification by flash column chromatography on silica gel (13\% to 67\% EtOAc in hexane) gave tetraol 11 (2.3 mg, 46\%) as an amorphous solid: ${ }^{1} \mathrm{H}$ NMR (600 MHz, CD $\left.3 \mathrm{OD}\right) \delta$ 6.23-6.27 (m, $2 \mathrm{H}$ ), 5.89 (dddd, $J=17.2,10.2,7.0,7.0 \mathrm{~Hz}, 1 \mathrm{H}$ ), 5.78 (s, $1 \mathrm{H}$ ), 5.38 (s, $1 \mathrm{H}$ ), 5.34 (s, $1 \mathrm{H}$ ), 5.20 (s, $1 \mathrm{H}$ ), 5.10 (dd, $J=17.2,1.5 \mathrm{~Hz}, 1 \mathrm{H}$ ), 5.05 (dd, $J=10.2,1.5$ Hz, 1 H), 4.97 (s, 1 H), 4.69 (dd, $J=6.6,4.8 \mathrm{~Hz}, 1 \mathrm{H}), 4.16$ (d, $J=4.4 \mathrm{~Hz}, 1 \mathrm{H}), 4.14$ (d, $J=4.4 \mathrm{~Hz}, 1$ H), 3.98 (d, $J=3.8 \mathrm{~Hz}, 1 \mathrm{H}), 3.70$ (ddd, $J=7.7,3.8,3.8 \mathrm{~Hz}, 1 \mathrm{H}), 3.06$ (dd, $J=16.4,4.5 \mathrm{~Hz}, 1 \mathrm{H}), 2.96$ (dd, $J=16.4,5.0 \mathrm{~Hz}, 1 \mathrm{H}$ ), 2.87 (dd, $J=6.7,2.0 \mathrm{~Hz}, 1 \mathrm{H}$ ), 2.71 (dd, $J=7.4,2.0 \mathrm{~Hz}, 1 \mathrm{H}$ ), 2.17-2.38 (m, 5 H), 2.29 (s, 3 H), 2.07-2.11 (m, 1 H), 1.22-1.32 (m, 5 H), 1.13 (d, $J=6.8$ Hz, 3 H), 0.99 (d, $J=6.2 \mathrm{~Hz}$, $3 \mathrm{H}), 0.85$ (t, $J=7.0 \mathrm{~Hz}, 3 \mathrm{H}) ;{ }^{13} \mathrm{C}$ NMR (150 MHz, CD $\left.3 \mathrm{OD}\right) \delta 168.4,155.8,151.9,149.4,137.5,137.5$, 
137.0, 119.1, 118.2, 115.0, 114.6, 83.6, 78.0, 76.7, 76.4, 75.5, 74.9, 71.9, 64.6, 59.0, 39.6, 38.4, 37.8, 37.6, 37.2, 22.9, 22.0, 18.4, 15.5, 15.4, 15.1; IR (KBr) $\quad v_{\max } 3437$ (br), 2958, 2353, 1716, $1150 \mathrm{~cm}^{-1}$; HRESIMS calcd for $\mathrm{C}_{31} \mathrm{H}_{46} \mathrm{O}_{7} \mathrm{Na} m / z$ 553.3141, found $m / z$ 553.3138; $[\alpha]_{\mathrm{D}}^{20}+6.7^{\circ}\left(c\right.$ 1.0, $\left.\mathrm{CH}_{2} \mathrm{Cl}_{2}\right)$.

\section{Diastereomer 2}

A solution of tetraol $11(4.7 \mathrm{mg}, 8.86 \mu \mathrm{mol})$ in DCE $(8.9 \mathrm{~mL})$ was degassed by F. T. P. The reaction mixture was heated to $50^{\circ} \mathrm{C}$ and $\mathrm{Cp} * \mathrm{Ru}(\mathrm{MeCN}){ }_{3} \mathrm{PF}_{6}(2.2 \mathrm{mg}, 4.4 \mu \mathrm{mol})$ was added. After $7 \mathrm{~h}$ at $50^{\circ} \mathrm{C}$, the reaction mixture was filtered through silica gel, and concentrated. Purification by $\mathrm{C}_{18}$ HPLC (Mightysil RP-18 250-4.6 (5 mm); eluent, 45\% CH3CN aq.; flow rate, $1.0 \mathrm{~mL} / \mathrm{min}$; UV detection at 265 $\mathrm{nm})$ afforded $2\left(0.96 \mathrm{mg}, 20 \%, t_{\mathrm{R}}=40.0 \mathrm{~min}\right)$.

2: ${ }^{1} \mathrm{H}$ NMR (600 MHz, $\left.\mathrm{C}_{6} \mathrm{D}_{6}\right) \delta 5.91$ (d, $\left.J=15.6 \mathrm{~Hz}, 1 \mathrm{H}\right), 5.91$ (s, $\left.1 \mathrm{H}\right)$, 5.72-5.81 (m, $\left.2 \mathrm{H}\right), 5.39$ (s, 1 H), 5.35-5.39 (m, 1 H), 5.29 (s, 1 H), 5.28 (s, 1 H), 4.97 (s, 1 H), 4.96-4.97 (m, 1 H), 4.90 (s, 1 H), 4.83 (s, $1 \mathrm{H}), 4.26$ (d, $J=4.3 \mathrm{~Hz}, 1 \mathrm{H}), 4.08$ (brs, $1 \mathrm{H}), 4.06$ (d, $J=4.3 \mathrm{~Hz}, 1 \mathrm{H}$ ), 3.86 (brs, $1 \mathrm{H}), 2.96$ (dd, $J=$ 7.1, $1.7 \mathrm{~Hz}, 1 \mathrm{H}), 2.93-2.97$ (m, $1 \mathrm{H}), 2.85$ (dd, $J=7.3,1.7 \mathrm{~Hz}, 1 \mathrm{H}), 2.67-2.71(\mathrm{~m}, 1 \mathrm{H}), 2.66$ (d, $J=7.5$ Hz, 2 H), 2.44 (dd, $J=14.1,5.2$ Hz, 1 H), 2.24-2.38 (m, 1 H), 2.28 (s, 3 H), 1.96 (dd, $J$ = 14.1, 9.1 Hz, 1 H), 1.27-1.42 (m, $5 \mathrm{H}), 1.17$ (d, $J=7.0 \mathrm{~Hz}, 3 \mathrm{H}), 1.01$ (d, $J=6.5 \mathrm{~Hz}, 3 \mathrm{H}), 0.86$ (t, $J=7.2 \mathrm{~Hz}, 3 \mathrm{H})$. ${ }^{1} \mathrm{H}$ NMR (600 MHz, $\mathrm{CDCl}_{3}$ ) $\delta 6.16$ (d, $\left.J=15.7 \mathrm{~Hz}, 1 \mathrm{H}\right), 6.08$ (ddd, $J=15.7,7.0,7.0 \mathrm{~Hz}, 1 \mathrm{H}$ ), 5.77 (s, $1 \mathrm{H}$ ), 5.69 (ddd, $J=15.4,7.6,7.6 \mathrm{~Hz}, 1 \mathrm{H}), 5.48$ (s, $1 \mathrm{H}$ ), 5.42 (dd, $J=15.4,5.1 \mathrm{~Hz}, 1 \mathrm{H}$ ), 5.39 (s, $1 \mathrm{H}$ ), 5.33 (s, 1 H), 5.20 (s, 1 H), 4.87 (s, 1 H), 4.79 (s, 1 H), 4.63 (dd, $J$ = 6.8, 3.0 Hz, 1 H), 4.38 (brs, 1 H), 4.18 (brs, 1 H), 4.14 (brs, 1 H), 3.92 (brs, 1 H), 3.21 (dd, $J=14.7,7.0$ Hz, 1 H), 2.98 (dd, $J=14.7,7.0$ Hz, 1 H), 2.88 (dd, $J=6.7,1.9$ Hz, 1 H), 2.71-2.76 (m, 2 H), 2.66-2.71 (m, 2 H), 2.50 (brs, 1 H), 2.47 (brs, $1 \mathrm{H}), 2.25-2.29$ (m, $2 \mathrm{H}), 2.25$ (s, $3 \mathrm{H}), 2.12-2.17$ (m, $1 \mathrm{H}), 1.89$ (dd, $J=14.0,9.2 \mathrm{~Hz}, 1 \mathrm{H}$ ), 
1.29-1.36 (m, $5 \mathrm{H}), 1.06$ (d, $J=7.0 \mathrm{~Hz}, 3 \mathrm{H}), 1.00$ (d, $J=6.4 \mathrm{~Hz}, 3 \mathrm{H}), 0.88$ (t, $J=7.0 \mathrm{~Hz}, 3 \mathrm{H}) ;{ }^{13} \mathrm{C}$ NMR (150 MHz, $\left.\mathrm{CDCl}_{3}\right) \delta 165.8,152.4,147.0,145.0,144.9,135.3,134.9,131.4,130.5,118.1,116.1$, 115.1, 113.1, 75.5, 75.4, 74.2, 73.5, 72.4, 62.3, 55.4, 40.0, 38.4, 36.3, 35.6, 35.3, 33.9, 20.1, 16.9, 14.9, 14.4, 14.2. ${ }^{1} \mathrm{H}$ NMR (600 MHz, CD $\left.{ }_{3} \mathrm{OD}\right) \delta 6.30$ (d, $\left.J=15.7 \mathrm{~Hz}, 1 \mathrm{H}\right), 6.21$ (ddd, $J=15.7,7.1,7.1 \mathrm{~Hz}, 1$ H), 5.84 (s, $1 \mathrm{H}), 5.56$ (ddd, $J=15.2,6.7,6.7 \mathrm{~Hz}, 1 \mathrm{H}), 5.41-5.44$ (m, $1 \mathrm{H}), 5.43$ (s, $1 \mathrm{H}), 5.38$ (s, $1 \mathrm{H}$ ), 5.27 (s, $1 \mathrm{H}), 5.13$ (s, $1 \mathrm{H}), 4.86$ (s, $1 \mathrm{H}), 4.77$ (s, $1 \mathrm{H}), 4.61$ (dd, $J=6.5,3.0 \mathrm{~Hz}, 1 \mathrm{H}), 4.22$ (d, $J=3.2$ Hz, $1 \mathrm{H}$ ), 4.11 (d, $J=3.2 \mathrm{~Hz}, 1 \mathrm{H}), 4.05$ (dd, $J=4.4,3.6 \mathrm{~Hz}, 1 \mathrm{H}), 3.87$ (d, $J=3.6 \mathrm{~Hz}, 1 \mathrm{H}), 3.24$ (dd, $J$ = 14.6, 7.1 Hz, $1 \mathrm{H}), 2.97-3.00$ (m, $1 \mathrm{H}), 2.96$ (dd, $J$ = 6.4, $2.1 \mathrm{~Hz}, 1 \mathrm{H}), 2.71$ (dd, $J=7.4,2.1 \mathrm{~Hz}, 1 \mathrm{H})$, 2.68-2.71 (m, $1 \mathrm{H}), 2.60$ (dd, $J=14.5,6.7 \mathrm{~Hz}, 1 \mathrm{H}), 2.34$ (d, $J=13.9,6.0 \mathrm{~Hz}, 1 \mathrm{H}), 2.26(\mathrm{~s}, 3 \mathrm{H})$, 2.10-2.16 (m, $1 \mathrm{H}), 1.93$ (dd, $J=13.9,8.0 \mathrm{~Hz}, 1 \mathrm{H}), 1.23-1.40$ (m, $5 \mathrm{H}), 1.08$ (d, $J=7.1 \mathrm{~Hz}, 3 \mathrm{H}), 0.99$ (d, $J=6.5 \mathrm{~Hz}, 3 \mathrm{H}), 0.89(\mathrm{t}, J=7.1 \mathrm{~Hz}, 3 \mathrm{H}) ;{ }^{13} \mathrm{C}$ NMR (150 MHz, $\left.\mathrm{CD}_{3} \mathrm{OD}\right) \delta 168.4,154.8,151.3$, 148.4, 148.0, 138.2, 137.1, 133.9, 131.9, 120.1, 116.1, 114.1, 113.7, 77.3, 76.6, 76.1, 75.4, 73.6, 64.3, 57.6, 41.9, 40.6, 38.8, 37.7, 37.5, 36.4, 22.0, 18.2, 16.5, 15.4, 15.4; IR (KBr) $v_{\max } 3423$ (br), 2925, 1634, $1151 \mathrm{~cm}^{-1}$; HRESIMS calcd for $\mathrm{C}_{31} \mathrm{H}_{46} \mathrm{O}_{7} \mathrm{Na} \mathrm{m} / \mathrm{z}$ 553.3141, found $\mathrm{m} / \mathrm{z} 553.3145$; $[\alpha]_{\mathrm{D}}{ }^{21}-11^{\circ}$ (c 0.6 , $\left.\mathrm{CHCl}_{3}\right)$.

\section{Acknowledgments}

We thank Professor B. M. Trost (Stanford University) for a generous offer of the sample of 3, and S. Oka, Center for Instrumental Analysis, Hokkaido University, for ESIMS measurements. This work was partly supported by a Grant-in-Aid for Scientific Research from the Ministry of Education, Science, Sports, and Culture of Japan. 


\section{References and Notes}

1) (a) Kobayashi, J.; Ishibashi, M.; Nakamura, H.; Ohizumi, Y.; Yamasu, T.; Sasaki, T.; Hirata, Y. Tetrahedron Lett. 1986, 27, 5755-5758. (b) Kobayashi, J.; Ishibashi, M.; Hirota, H. $\quad$ J. Nat. Prod. 1991, 54, 1435-1439.

2) Lam, H. W.; Pattenden, G. Angew. Chem. Int. Ed. 2002, 41, 508-511.

3) Maleczka, R. E. Jr.; Terrell, L. R.; Geng, F.; Ward, J, S., III Org. Lett. 2002, 4, 2841-2844.

4) (a) Trost, B. M.; Chisholm, J. D.; Wrobleski, S. T.; Jung, M. J. Am. Chem. Soc. 2002, 124, 12420-12421. (b) Trost, B. M.; Harrington, P. E. J. Am. Chem. Soc. 2004, 126, 5028-5029. (c) Trost, B. M.; Wrobleski, S. T.; Chisholm, J. D.; Harrington, P. E.; Jung, M. J. Am. Chem. Soc. 2005, 127, in press (DOI: 10.1021/ja0533646). (d) Trost, B. M.; Harrington, P. E.; Chisholm, J. D.; Wrobleski, S. T. J. Am. Chem. Soc. 2005, 127, in press (DOI: 10.1021/ja053365y).

5) Assignments of some carbon chemical shifts for synthetic compound $\mathbf{1}$ by Trost's group were equivocal, while there were no assignments for them by Pattenden’s group.

6) Kita, Y.; Maeda, H.; Omori, K.; Okuno, T.; Tamura, Y. Synlett 1993, 273.

7) Trost, B. M.; Toste, F. D. J. Am. Chem. Soc. 2000, 122, 714

8) Ketal groups were difficult to remove at final stage.

9) Kita's method ${ }^{6}$ does not require MS3A, but the reaction yield was low without MS3A.

10) Esterification under Keck and Mitsunobu conditions gave a complex mixture. 


Asymmetric Synthesis of a Diastereomer of
Structure Proposed for Amphidinolide A and the
determination of Its Absolute Configuration
Haruaki Ishiyama, Yoichi Nakamura, and Jun'ichi Kobayashi*
Graduate School of Pharmaceutical Sciences, Hokkaido University, Sapporo 060-0812, Japan this area blank for abstract info.
Asymmetric synthesis of a diastereomer (2) of the structure (1) proposed for
amphidinolide A, a cytotoxic macrolide from the cultured dinoflagellate
Amphidinium sp., has been accomplished. Absolute configuration of
amphidinolide A was concluded to be 3 from comparison of spectral data of
amphidinolide A and synthetic diastereomers 2 and 3, the latter of which
was synthesized by Trost's group.

